\title{
MISURA DELLA RADIOATTIVITÀ ATMOSFERICA CON EMULSIONI NUCLEARI
}

\author{
E. Moraschinelli
}

E noto che le sostanze radioattive contenute nell'aria si trovano in gran parte ancorate alle particelle che costituiscono l'aerosol atmosferico. Per la misura della radioattività atmosferica si ricorre perciò sovente alla separazione di tali sostanze da volumi noti di aria, e poi alla misura della loro attività con uno dei metodi che sono normalmente impiegrati per le sostanze solide.

La scparazione può essere ottenuta sia mediante aspirazione dell'aria altraverso filtri che trattengono le particelle solide di dimensioni superiori ad un certo limite ${ }^{1}{ }^{1}$, sia con la precipitazione elettroslatica in una scarica ad effuvio fra un elettrodo a punta e uno piano (-). Di questo secondo metodo fa uso l'apparecchio di G. Aliverti per la misura quantitativa della radioattività dell'aria $\left({ }^{3}\right)$.

L'apparecchio che viene qui descritto è hasato sullo stesso principio e in un certo senso si può ritenere una modifica di questo, resa possilile dalla recente produzione di lastre fotografiche adatte per misure di radioattività.

Un volume noto di aria, aspirato a velocità costante altraverso l'ap. parecchio, viene privato delle particelle solide alle quali sono ancorate le sostanze radioattive. Con il metodo Aliverti l'esame della radioattivilà del deposito oltenuto viene eseguito misurando con una camera di ionizzazione il decadimento della sua attività; in questo caso invece, ponendo il deposito in contatto con l'emulsione sensibile di una lastra nucleare si ha una registrazione delle particelle $u$ emesse durante il suo decadimenio. Dopo lo sviluppo dell'emulsione il suo esame microscopico permette di determinare la quantità $e$, approssimativamente, anche la natura delle sostanze depositate.

Con questo metodo si possono ottenere praticamente soltanto valori relativi della radioattività atmosferica; però questi sono i soli che interessano quando si voglia studiare l'andamento di tale radioattività col tempo, o in diversi luoghi. La misura consiste nel contare le tracce su una limitata superficie dell'emulsione, scelta in modo opportuno. 
L'impiego di lastre nucleari per la misura della radioattivita atmosferica presenta alcuni vantaggi rispetto agli altri metodi comunemente impiegati. Trattandosi di attivita molto piccole, la proprieta integratrice dell'emulsione permetle di ottenere una maggior precisione nella misura. Inoltre, osservando l'emulsione al microscopio, è facile riconoscere fra le tracce quelle prodotte dalla disintegrazione del $T h_{l} C^{\prime}$ che hanno una lunghezza di $46,6 \mu\left({ }^{4}\right)$, mentre le altre arrivano al massimo a 38.4 u ( $R a C^{\prime}$. Questo fatto permetle li valutare approssimativamente il contenuto di elementi della famiglia del torio nell'atmosfera.

Infine, vantagrio non trascurabile, l'apparecchio può essere facilmente trasportato da un luogo all'altro per rilievi radioattivi, poiché le lastre impiegate nella misura possono essere conservate per lo sviluppo e l'osservazione in un secondo tempo.

Apporecchio. - L'aria da esaminare viene aspirata con un aspiratore, azionato da un motorino elettrico o a molla, attraverso un tuloo di materiale isolante (plexiglasi della lungrhezza di $25 \mathrm{~cm}$ e di sezione rettangolare di $1,5 \times 2.5 \mathrm{~cm}$. Nel raccordo fra questo tubo e l'aspiratore $\dot{e}$ inserito un anemometro, che permette di determinare la quan. lità di aria fluita attraverso l'apparecchio.

lungo il tubo, a 5 e a $15 \mathrm{~cm}$ dall'ingresso, sono posti due precipitatori, costituiti ognumo da una fila di 5 punte di acciaio che entrano altraverso fori praticati traversalmente in una parete, penctrando per 5 mm nell'interno. Di fronte ad esse, nella parete opposta è praticata un'apertura di $2,5 \times 2,5$ cm nella quale viene posta una lastrina di ottone, in modo di chiuderla ermeticancnte.

$\mathrm{Si}$ redrà più avanti cone la funzione del secondo precipitatore, a valle del primo, sia quella di permettere la determinazione dell'efficienza del primo nella precipitazione delle sostanze radioattive, e di fornire cosi una taratura dell'apparecchio, indispensabile per eseguire le misure.

Le lastrine di ottone che fungono da elettrodo piano dei precipitatori sono fissate con una molla, e possono essere facilmente sostituite: di queste hisogna disporre una certa riserva, il cui numero dipende dal programma di misure che si vogliono eseguire.

Le lastre fotografiche vengrono conservate in una scatola di legno a tenuta di luce. Qualsiasi tipo di emulsione sensibile alle particelle

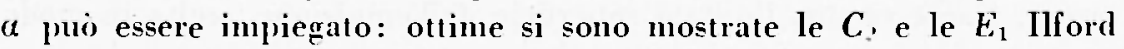
con emulsione da $50 u$ e dimensioni $2,5 \times 12,5 \mathrm{~cm}$. Esse vanno pre- 
disposte nella cassetla con l'emulsione rivolta in posizione facilmente riconoscibile. Quando si è ottenuto il precipitato radioattivo su un clettrorlo, si porta questo nel più breve tempo possibile, e comunque: sempre uguale, in contatto con l'emulsione, alla quale si mantiene aderente con un elastico di gomma, o con una molletta di acciaio.

Se le misure vengono fatte in campagna, questa operazione puì essere fatta mantenendo la scatola delle lastre in una custodia di panno nero, nella quale si infilano le mani per eseguire tutte le operazioni senza esporre alla luce le emulsioni sensibili.

Per la precipitazione si applica agli elettrodi del precipitatore una differenza di potenziale di alcune migliaia di volt. La velocità di precipitazione è tanto maggiore quanto più questo potenziale è elevato, ed è massima quando si fa uso di tensione continua con punte negative". Ad es. un deposito sufficiente per fare una misura si ottiene, con un flusso di aria di l litro/min e tensione alle punte di 10 kVolt, con una precipitazione della durata di 15 minuli.

In campagna il precipitatore può essere alimentato con un generatore portatile a pile e oscillatore elevatore di tensione". Se invece si ha a disposizione la corrente alternata della rete, un piccolo alimentatore, costituito da un trasformatore del tipo impiegato negli impianti per insegne luminose da 10 watl, $6 \div 7 \mathrm{kVolt}$, seguito da un diodo raddrizzatore (es. 1B3 Firre) e da un condensatore di livellamento, può fornire la tensione continua richiesta.

Misura. - Messo in funzione l'aspiratore, si chiude il circuilo che dà la tensione al precipitatore per la durata desiderata; poi si toglie l'clettrodo piano e lo si appogria alla lastra fotografica, lasciandovelo un tempo sufficiente per permettere il decadimento completo dell'altività del deposito. Poiché le sostanze radioattive contenute nell'almosfera sono limitale, almeno quelle naturali, ai prodotti di disintegrazione delle emanazioni di radio e di torio, hasta tener conto del più lungo dei periodi di lali sostanze, che è quello del $T h B$, di 10.6 ore.

Il completo decadimento del $T h B$ e dei suoi prodotti successivi in cquilibrio transiente con esso, aviene praticamente in un intervallo di $40 \div 50$ ore. Le lastre devono essere percio lasciate a contatto dell'elettrodo almeno due griorni prima di procedere allo sviluppo: questo viene poi eseguito secondo le istruzioni della Ilford, e non offre particolari difficolı̀. 
Per contare le tracce delle particelle a nell'emulsione si usa un microscopio munito di tavolino traslatore, con un ingrandimento di $400 \div 500 x$. La discriminazione delle particelle $u$ del $T h C^{\prime}$ può essere fatta ricorrendo alla loro misura con un oculare contenente una scala tarala. Naturalmente col microscopio si osservano le proiezioni orizzontali delle tracce; le più inclinate nell'emulsione vengono senz'altro escluse da questa classificazione a parte. Però si vedrà più avanti come il numero delle tracce con componente orizzontale tale da poter essere attribuite al $T h C^{\prime}$ sia sufficiente per una valutazione approssimata del contenuto di questo elemento nel deposito.

Taratura dell'apparecchio. - La laratura permelle di stabilire quale percentuale di portatori radioattivi viene separata dall'aria che fluisce attraverso l'apparecchio. Questa percentuale dipende dalle condizioni di funzionamento, cioè dalla tensione usata per la precipitazione e dalla velocità di aspirazione dell'aria; la taratura deve perciò essere rifatta ogni volta che vengono cambiati questi parametri.

Poiché in pratica è facile mantenere costante la lensione al precipitatore, mentre la velocità di aspirazione che dipende, fra l'altro, anche dalla pressione atmosferica, può risultare diversa da una misura all'altra, è conveniente tracciare preventivamente una curva di taratura, come suggerito da G. Aliverti ${ }^{3}$. Per un determinato valore della tensione al precipitatore, si valuta, col melodo indicato più sotto, la percentuale dei portatori radioattivi precipitati $X$, in funzione della velocita di aspirazione $V$. Entro ampie variazioni della velocita, questa funzione ̀̀ rappresentata da una relta, che può essere tracciata con la misura di due sole coppie di valori di $\mathrm{X}$ e $\mathrm{V}$.

L'operazione di taratura viene eseguita collegando in parallelo $\mathrm{i}$ due precipitatori e impressionando due lastre con i depositi ollenuti simultaneamente. Il contegrio delle tracce su una certa zona dell'emul. sione della seconda lastra (quella corrispondente al precipitalore a valle), darà un numero $n$. di tracce per unità di superficie inferiore alle $n_{1}$ della prima lastra. 11 calcolo di $X$ si esegue nel sequente modo. Sia $N$ il numero di portatori radioattivi per $\mathrm{cm}^{3}$ di aria; il primo precipitatore ne deposita $N X=k_{i} n_{1}$. Al secondo precipitatore giunge aria contenente soltanto $N(1-X)$ portatori per $\mathrm{cm}^{3}$ : di questi ne vengono precipitati $N(1-X) X=k n$. Dal rapporto fra queste due relazioni si ricava il valore di $X$ :

$$
X=1-n_{2} / n_{1}
$$


La costante $k$ è uguale per le due lastre se si ha cura di scegliere per l'esplorazione una superficie di emulsione situata in posizione corrispondente rispetto alle punte dei precipitatori.

Contenuto relativo di elementi della famiglia del torio. - Mediante la misura della lunghezza delle tracce nell'emulsione è possibile costruire uno spettro dei percorsi che permette di riconoscere sli elementi presenti nel deposito e ricavare la quantità relativa di ciascuno di essi ${ }^{7}$. Questa operazione è alquanto laboriosa, perché la lunghezza di ogni traccia deve essere ricavala misurando la sua proiezione orizzontale e la sua penetrazione nell'emulsione, mettendo a fuoco successivamente il primo e l'ultimo grano di essa, e correggendo la componente verticale per tener conto della contrazione della gelatina dopo lo sviluppo.

Come si è visto, il maggior percorso nell'emulsione delle particelle a del $T h C^{\prime}$ permette di riconoscere le loro tracce e valutare approssimativamente il contenuto relativo di $T h C^{\prime}$, e quindi di $T h C$ e $T h B$, nel deposito.

Poiché l'apparecchio descritto serve per misure relative della radioatlivita dell'aerosol atmosferico, la percentuale di tracce con componente orizzontale di lunghezza maggiore di 38,4 u già per se stessa $\therefore$ sufficiente per seguire l'andamento del rapporto fra gli elementi delle due famiglie nell'atmosfera. Lo stesso rapporto per gli elementi presenti nel deposito alla fine della precipitazione, può essere calcolato determinando prima il numero delle tracce di $T h C^{\prime}$ nell'emulsione. G. Imbò e L. Casertano ${ }^{8}$ e J. H. Poole e C.M.E. Mattews ${ }^{9}$ hanno risolto il problema di calcolare il numero di tracce di un dato percorso conoscendo il numero di quelle con proiezione orizzontale maggiore di un certo limite. In questo caso, trattandosi di un deposito radioaltivo infinitamente soltile, tale cioè da non assorbire parte dell'energia delle particelle emesse nello spessore stesso del deposito, il calcolo risulta particolarmente semplice.

Se $\theta$ è l'angolo compreso fra la direzione di una traccia e la sua proiezione nel piano della gelatina, all'osservazione microscopica vengono riconosciute come tracce del $T h C^{\prime}$ soltanto quelle che penetrano nell'emulsione con un angolo inferiore a $34^{\circ} 30^{\prime}$. Infatti la condizione per la discriminazione è :

$$
46,6 \cos \theta>38,4 \text { da cui } \theta<34^{\circ} 30^{\prime}
$$


Sia $N^{\prime}$ il numero delle tracce discriminate su un totale $N^{\prime \prime}$ di tracce contate; le tracce del $T h C^{\prime}$ saramno $N^{\prime} P$ dove $P$ è il rapporto tra $2 \pi$ e l'angolo solido con generatrici $\theta=0$ e $\theta=34^{\circ} 30^{\prime}: P=1,76$.

Il numero $N^{\prime \prime}$ - $N^{\prime}$ delle tracce con lunghezza inferiore a 38,4 u deve essere corretto per tener conto di quelle tracce che sfuggono all'osservazione perché hanno $\theta$ uguale o vicino a $90^{\circ}$. Per questa correzione è stato determinato sperimentalmente un fattore $K=1,15$.

Il numero delle tracce del $T h C^{\prime}$ sta a quelle del $T h C$ nel rapporto costante $66,3 / 33,7$. Il $T h B$ emelte una particella $\beta$ nella trasformazione $T h B \stackrel{\beta}{\longrightarrow} T h C$ e successivamente una particella $\alpha$ nella trasformazione $T h C \stackrel{\alpha}{\longrightarrow} T h C^{\prime \prime}$ oppure $T h C \stackrel{\stackrel{\rho}{\longrightarrow}}{\longrightarrow} T h C^{\prime} \stackrel{\text { ih }}{\longrightarrow} T h D$ : perciò ogni elemento delle due serie si trasforma in elemento stabile con l'emissione di una sola particella $\alpha$, ad eccezione del $R a A$, che potrebbe dare nell'emulsione due tracce aventi un punto di emissione comune. Tracce di questo tipo non sono state osservate nelle misure fatte finora con questo apparecchio, e anche in lavori analoghi (i.11) è stata notata la loro mancanza. Il rapporto $R$ fra gli elementi della famiglia del torio $(T h B+T h C$ ) e quelli della famiglia del radio presenti nel deposito alla fine della precipitazione è dato da:

$$
R=N^{\prime} P(1+33,7 / 66,3) /\left[\left(N^{\prime \prime}-N^{\prime}\right) K+N^{\prime}+N^{\prime} \mathrm{P}(1+33,760.31]\right.
$$

Per risalire al valore di questo rapporto nell'atmosfera, bisognerebbe supporre, come si fa generalmente, che gli elementi delle due serie si trovino sull'aerosol in equilibrio radioattivo fra loro. Dai risultati di ricerche eseguite in questo Istituto con vari metodi ${ }^{10}$, sembra che questa condizione non sia verificata per le sostanze ancorate all'aerosol e precipitate elettrostaticamente; come conseguenza il valore di $\bar{R}$ calcolato risulterebbe affetto da un errore difficilmente valutabile.

Colgo l'occasione per ringraziare il Prof. O. Specchia, direttore dell'Istituto di Fisica dell'Università di Pavia, per l'interesse mostrato per questo lavoro, e la Prof. G. Aliverti per i preziosi consigli e le discussioni sull'argomento.

Pavia - Istituto di Fisica dell'Università - Luglio 195.3. 


\section{RIASSUNTO}

Viene descritto un metodo per la misura della radioattività atmosferica con l'impiego di lastre fotografiche per ricerche nucleari. 'Le sostanze radioattive vengono separate dall'aria mediante precipitazione elettrostatica su un olettrodo piano, il quale viene in seguito appoggiato sull'emulsione sensibile, per ottenere una registrazione delle particelle a emesse. L'esame microscopico dell'emulsione permette di determinare il valore relativo della radioatività atmosferica e di discriminare gli elementi della famiglia del torio da quelli della Jamiglia dell'uranio-rudio.

\section{$S L M M A R Y$}

A method of measuring atmospheric radioactivity by the employment of nuclear emulsions is described. The radioaclive components are separated from the air by electrostatic separation into an electrode plate, folloning which, the plate is placed on the sensitive emulsion in order to obtain a registration of the emilted alpha particles. From a microscopic examination of the emulsion one determines the relative amount of the atmospheric radioactivity and it is also possible to discriminate the olements of the thorium family from those of the uranium-radium iamily.

\section{BIBLIOGRAFIA}

(1) J. H. Hard.EY: Nucleonics 11, 12, (luglio 1953),

( $\because$ A. Skll1: Rend. Acc. Lincei, XI, 58, 212, (1902).

(i) G. Aliviltt: N. Cim., 8, 233, 11931); N. Cim., 9, 313, (1932).

(1) J. P. Lovecunup: J. Phys. Rad., 11, 89. (1953).

(5) G. Arivelitı: N. Cim., 12, 26, 1935): E. Momaschnvelda: Rend. Ist. Lomb., $86,53,(1953)$.

(9) E. MonuscunvidrI: Rend. Ist. Lomb., 86, 281, (1953).

(7) E. Molaschinet.r.1: N. Cinı., 10, 1341, (1953).

(8) G. Imò, L. Casfantaxo: Ann. Geof., 3, 475, (1950); 5, 1, 1952).

(9) J. H. Pool.f, C. M. E. Mitrews: Sci. Proc. Roy. Dublin Soc., 25, 305, (1951).

(10) E. Morascmivfld1: Rend. Ist. Iomb., 84, 293, (1951); N. Cim., 10, 1341, (1953).

(11) R. Sikssa: Ark. Geophys., 1 (6). 123, (1950). 